\title{
0 papel dos modificadores de rede na produção da fotoluminescência no $\mathrm{CaWO}_{4}$
}

\section{(The role of network modifiers in the production of photoluminescence in $\mathrm{CaWO}_{4}$ )}

\author{
F. R. C. Ciaco ${ }^{1}$, F. M. Pontes ${ }^{1}$, C. D. Pinheiro ${ }^{1,2}$, E. R. Leite ${ }^{1 *}$, S. R. de Lazaro', J. A. Varela ${ }^{3}$, P. S. \\ Pizani $^{4}$, C. A. Paskocimas ${ }^{3}$, A. G. Souza ${ }^{5}$ E. Longo ${ }^{1}$ \\ ${ }^{1}$ CMDMC-LIEC, Departamento de Química, Universidade Federal de S. Carlos \\ Rod. Washington Luiz km 235, C.P. 676, S. Carlos, SP, 13565-905 \\ ${ }^{2}$ DCEN, CFP, Universidade Federal da Paraíba, Cajazeiras, PB \\ ${ }^{3}$ Instituto de Química, UNESP \\ C.P. 355, Araraquara, SP, 14801-970 \\ paskocimas@liec.ufscar.br \\ ${ }^{4}$ Departamento de Física, Universidade Federal de S. Carlos \\ Rod. Washington Luiz km 235, C.P. 676, S. Carlos, SP, 13565-905 \\ ${ }^{5} D Q$, CCEN, Universidade Federal da Paraíba, J. Pessoa, PB
}

\begin{abstract}
Resumo
Discutiu-se a fotoluminescência para o tungstato de cálcio amorfo, observada à temperatura ambiente. Verificou-se que há concordância entre os resultados experimentais e teóricos. Neste trabalho foram simuladas as estruturas cristalinas e amorfas do tungstato de cálcio $\left(\mathrm{CaWO}_{4}\right)$, comparando-se as respectivas estruturas eletrônicas. Os resultados dos cálculos teóricos indicam a formação de novos níveis de energia na banda de valência e de condução do amorfo. Estes níveis eletrônicos extras são os responsáveis pela formação da cauda na curva do espectro de absorção. Correspondentemente, medidas experimentais de absorção óptica mostraram a presença da cauda, com relação ao espectro do cristalino. Desta forma, pode-se interpretar a formação da cauda, como sendo associada aos defeitos promovidos na estrutura desordenada do material amorfo.

Palavras-chave: fotoluminescência, processamento químico, mecânica quântica.
\end{abstract}

\begin{abstract}
We discuss the nature of visible photoluminescence at room temperature in amorphous calcium tungstate taking into account the results of recent experimental and quantum mechanical theoretical studies. Our investigation of the electronic structure involved the use of first-principle molecular calculations to simulate the variation of the electronic structure in the calcium tungstate crystalline phase, which is known to have a direct band gap, and we also made an in-depth examination of amorphous calcium tungstate. The results of our theoretical calculations of amorphous calcium tungstate indicate that the formation of threefold coordination in the amorphous system may introduce delocalized electronic levels in the HOMO (highest occupied molecular orbital) and the LUMO (lowest unoccupied molecular orbital). These delocalized electronics levels are related to the formation of a tail in the absorbency spectrum curve. The results indicate that amorphous calcium tungstate has the conduction band near the band gap dominated by Ca states contribution. Experimental optical absorption measurements showed the presence of a tail. These results are interpreted by the nature of these exponential optical edges and tails, associated with defects promoted by the disordered structure of the amorphous material. We associate them with delocalized states in the band gap.
\end{abstract}

Keywords: photoluminescence, chemical processing, quantum mechanics.

\section{INTRODUÇÃO}

O desenvolvimento de materiais semicondutores, com propriedades ópticas ativas como fotoluminescência (FL), eletroluminescência, ou propriedades ópticas não lineares pode conduzir a novos dispositivos com desempenho superior ou a diferentes modos de atuação $[1,2]$. Neste sentido, tem havido atualmente um interesse muito grande, no estudo da FL, em materiais amorfos ou materiais não estruturados. Isso é confirmado pelo intenso esforço de pesquisa, que vem sendo feito no estudo de vários tipos de FL visível, em temperatura ambiente, como por exemplo no caso do silício poroso [3]. Durante a década passada, os compostos luminescentes foram extensivamente estudados, sendo que estes estudos envolveram 
amostras cristalinas puras ou dopados com terras raras, tais como os íons $\mathrm{Eu}^{3+}, \mathrm{Nd}^{3+}, \mathrm{Er}^{3+}$ e $\mathrm{Mn}^{4+}$. Estes sistemas foram abordados devido ao seu potencial em aplicações ópticoeletrônicas [4]. A FL foi estudada à baixa temperatura $(\mathrm{T}=15 \mathrm{~K})$ em cristais de $\mathrm{SrTiO}_{3}$ dopados com manganês e sob excitação com laser de $532 \mathrm{~nm}$ [5]. O comportamento luminescente do íon $\mathrm{Mn}^{4+}$, como impureza na estrutura perovsquita $\mathrm{ABO}_{3}$, foi analisado. Por outro lado, a FL foi estudada a 10K em PLZT dopado com $\mathrm{Eu}^{3+}, \mathrm{Nd}^{3+}$ e $\mathrm{Cr}^{3+}$ [6]. Em ambos trabalhos foram observados centros luminescentes, e argumentado que os mesmos correspondem a um processo de transferência de carga, envolvendo um sistema de buraco (h) e elétron (e).

Desta forma, em materiais cristalinos a baixas temperaturas, a fotoluminescência tem sido associada com a excitação dos grupos tetraédricos $\mathrm{WO}_{4}$. No entanto, compostos amorfos do tipo tungstato com FL, em temperatura ambiente, ainda não tinham sido observados. Neste caso, as propriedades ópticas dos compostos semicondutores amorfos são caracterizadas pela presença de uma cauda no espectro de absorção óptica, na região em que normalmente o sólido cristalino é transparente [7-9]. Este comportamento é denominado absorção óptica da cauda quase zero. O chamado limite de Urbach é atribuído à presença de estados eletrônicos localizados próximos ao limite do "band-gap" do semicondutor amorfo. Materiais com estas características ópticas podem apresentar estas propriedades FL $[10,11]$. Como no caso dos tungstatos amorfos, várias propriedades interessantes de perovsquitas têm sido relatadas, inclusive mostrando que o comprimento de onda emitido está relacionado ao comprimento de onda incidente [12-16].

Neste artigo, foram sintetizadas amostras do $\mathrm{CaWO}_{4}(\mathrm{CW})$, amorfas e cristalinas, e investigadas as suas propriedades fotoluminescentes. Por outro lado, foram analisadas as propriedades ópticas utilizando métodos mecânico-quânticos a nível ab-inítio, para melhor compreensão dos fenômenos observados.

\section{MATERIAIS E MÉTODOS}

Os pós de $\mathrm{CaWO}_{4}$, amorfo e cristalino, foram sintetizados pelo método dos precursores poliméricos [15-16]. Este método tem sido usado, com grande sucesso, para sintetizar nanopartículas e filmes finos de vários sistemas complexos de óxidos policatiônicos. O processo baseia-se na solubilização dos cátions de interesse, normalmente em solução aquosa, e na subseqüente quelação destes cátions por um ácido carboxílico, normalmente ácido cítrico. A solução de citrato é misturada com um glicol, preferencialmente etilenoglicol, para promover a polimerização por intermédio da reação de poliesterificação. Essa reação ocorre em temperaturas entre 90 e $120^{\circ} \mathrm{C}$, sendo favorecida pela redução da concentração de água. Na etapa seguinte, o precursor polimérico é calcinado a $300{ }^{\circ} \mathrm{C}$, para promover a pré-pirólise. Otimizando-se o tempo de tratamento térmico, promove-se a oxidação completa da matéria orgânica, sendo que, o uso de atmosfera de oxigênio acelera o processo de eliminação do carbono, proveniente da síntese. Este procedimento foi adotado para obtenção do material amorfo. Em seguida, uma parte do material amorfo foi tratado termicamente a $600{ }^{\circ} \mathrm{C}$ por $2 \mathrm{~h}$, para obtenção do material cristalino.

As medidas de fotoluminescência foram feitas em um equipamento V1000 Jobin-Yvon com monocromador duplo
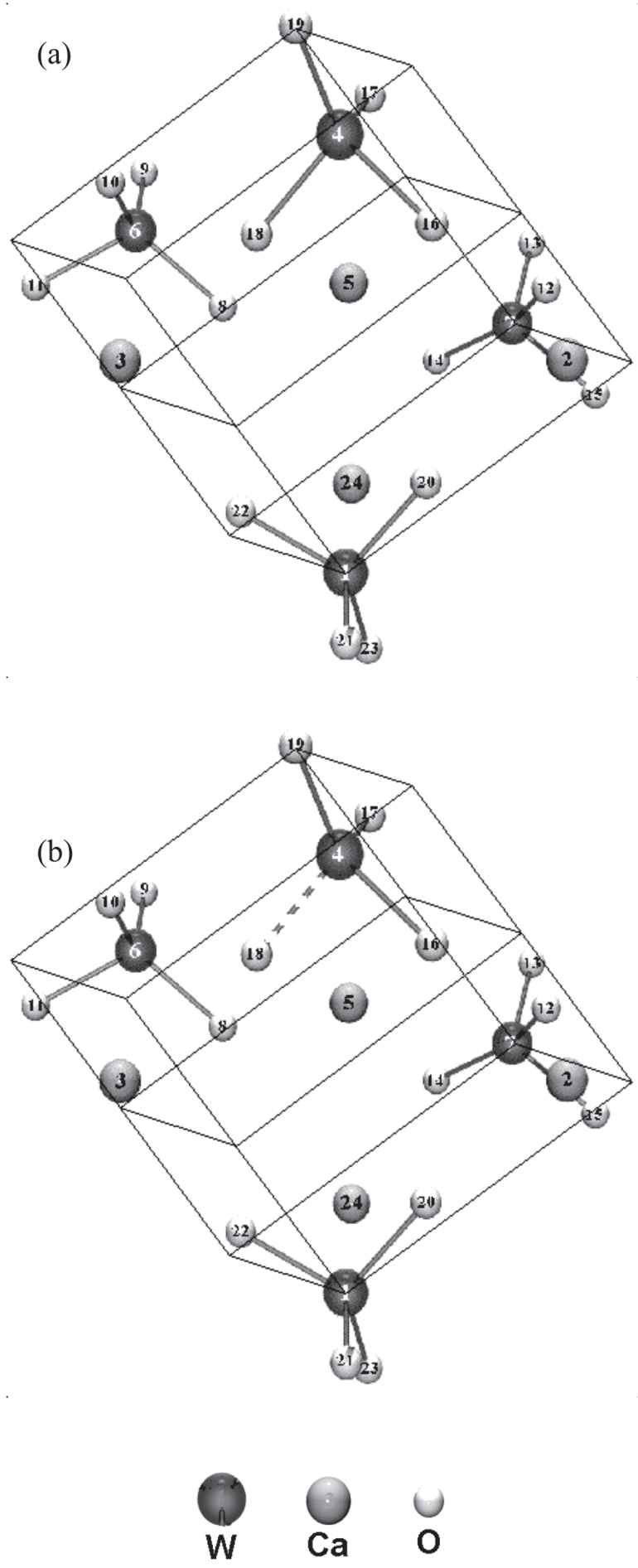

Figura 1: Representação esquemática do "cluster" do $\mathrm{Ca}_{4} \mathrm{~W}_{4} \mathrm{O}_{16}$, (a) cristalino e (b) amorfo.

[Figure 1: Schematic representation of the "cluster" of $\mathrm{Ca}_{4} \mathrm{~W}_{4} \mathrm{O}_{16}$ (a) crystalline "cluster" and (b) amorphous "cluster".] 
acoplado a uma fotomultiplicadora de cianeto de gálio conectada a um sistema contador de fótons. As amostras foram submetidas a um laser de íons argônio, com comprimento de onda de 488,0 nm, e potencial de saída máxima em torno de $20 \mathrm{mV}$; as medidas foram realizadas à temperatura ambiente.

Os pós de $\mathrm{CW}$ foram caracterizados estruturalmente por difração de raios X em um difratômetro Siemens D5000. Os espectros de absorção óptica para o $\mathrm{CaWO}_{4}$, cristalino e amorfo, foram obtidos à temperatura ambiente em um espectrofotômetro Cary 5G.

Os dados de espectroscopia Raman foram obtidos em um equipamento RFS/100/S Bruker FT-Raman, com resolução espectral de $4 \mathrm{~cm}^{-1}$, equipado com laser de $\mathrm{Nd}$ :YAG, promovendo uma excitação de luz em $1064 \mathrm{~nm}$. Foi investigada a faixa espectral de 100 até $1000 \mathrm{~cm}^{-1}$; todas as medidas foram feitas à temperatura ambiente.

Os cálculos teóricos consideraram as variações na estrutura eletrônica do $\mathrm{CaWO}_{4}$ entre duas configurações: tetracoordenadas e tri-coordenadas. Nesta simulação foram adotados dois "clusters" para representar a estrutura cristalina, conforme representado na Fig. 1a e na Fig. 1b. Os cálculos em nível ab-initio foram realizados utilizando os seguintes conjuntos de bases atômicas: o oxigênio e o cálcio (6-31Gd) e o tungstênio do pseudopotencial do tipo LanL2DZ [17-20]. Para realização dos cálculos, foi utilizado o pacote computacional Gaussian98 [21].

Foram calculadas as densidades de estados e o "gap" para os "clusters" no estado amorfo e no cristalino. Por outro lado, as cargas atômicas foram computadas utilizando-se orbitais naturais (NBO). Nesta aproximação, a estrutura do $\mathrm{WO}_{4}$, tetracoordenada, é representada pela Fig. 1a, derivada a partir dos dados cristalográficos. A estrutura tetracoordenada foi otimizada e depois deformada de 1,0 A. Para tanto foi aplicada uma deformação na ligação entre os átomos W(4)-O(18), obtendo-se uma estrutura tricoordenada, associada a uma tetracoordenada conforme ilustrada na Fig. 1b. Deve-se salientar que a banda de valência superior é associada ao orbital molecular ocupado mais alto (HOMO) e a banda de condução ao orbital molecular desocupado mais baixo (LUMO).

\section{RESULTADOS E DISCUSSÃO}

Os resultados de análise termogravimétrica, realizados na isoterma de $300{ }^{\circ} \mathrm{C}$, por um período de $20 \mathrm{~h}$, permitiram certificar que os pós sintetizados não apresentam perda de massa posterior. A Fig. 2 ilustra a difração de raios $\mathrm{X}$ dos pós de $\mathrm{CaWO}_{4}$ calcinados a diferentes temperaturas. As amostras calcinadas em baixa temperatura mostraram um padrão de difração característico de estruturas amorfas, enquanto que o material tratado a $600{ }^{\circ} \mathrm{C}$ apresentou um padrão de difração característico de estruturas cristalinas. Neste caso, os picos de difração foram associados à estrutura ortorrômbica. A Fig. 3 ilustra o comportamento da FL observada nos pós amorfos e cristalinos, com uma excitação de comprimento de onda 488,0 nm à temperatura ambiente. A FL no material amorfo mostrouse intensa, observando-se luminescência na região visível com um pico máximo em $600 \mathrm{~nm}$. Os espectros apresentados na

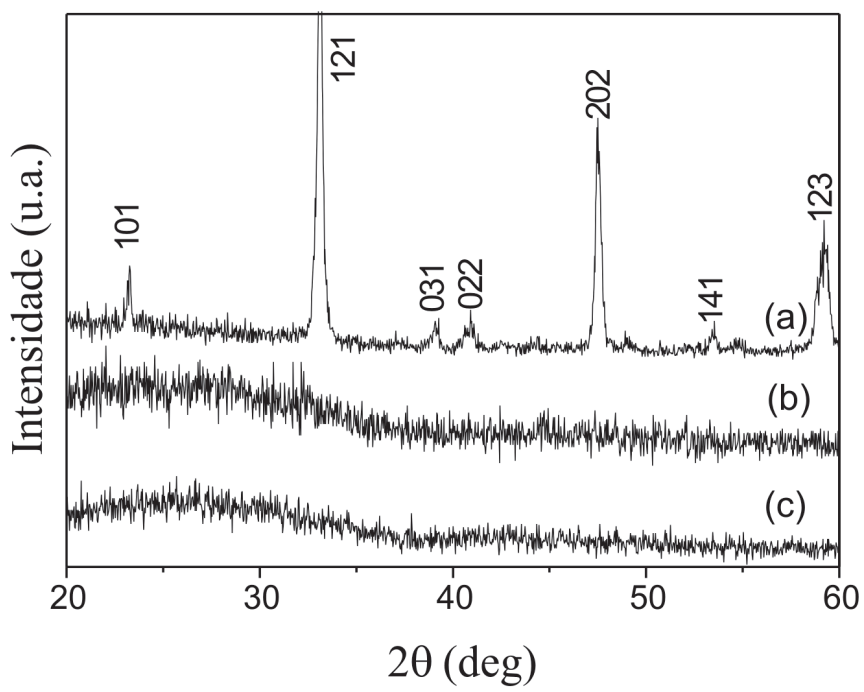

Figura 2: Padrão de difração de raios X do pó de CaWO4 calcinado em condições variadas de temperatura sob fluxo de oxigênio: (a) cristalino, $600{ }^{\circ} \mathrm{C} / 2 \mathrm{~h}$; (b) amorfo, $300{ }^{\circ} \mathrm{C} / 12 \mathrm{~h}$; (c) amorfo, $300^{\circ} \mathrm{C} / 32 \mathrm{~h}$.

[Figure 2: XRD patterns of CaWO4 powders calcined at different temperatures under oxygen flow: (a) Crystalline, $600{ }^{\circ} \mathrm{C} / 2 \mathrm{~h}$; (b) amorphous, $300{ }^{\circ} \mathrm{C} / 12 \mathrm{~h}$; (c) amorphous, $\left.300^{\circ} \mathrm{C} / 32 \mathrm{~h}.\right]$

Fig. 3 ilustram a dependência da FL, em relação às condições de tratamento térmico, caracterizando a influência da temperatura na organização do material amorfo e como decorrência uma diminuição da FL. A influência de carbono na FL do material CW foi eliminada pelo monitoramento das amostras por análise térmica. Assim sendo, a redução da quantidade de carbono no pó foi analisada por termogravimetria até a condição de completa eliminação deste resíduo. Desta forma, elevando a temperatura ou aumentando o tempo de tratamento térmico, existe um decréscimo na quantidade de carbono total. Assim sendo, à baixa temperatura

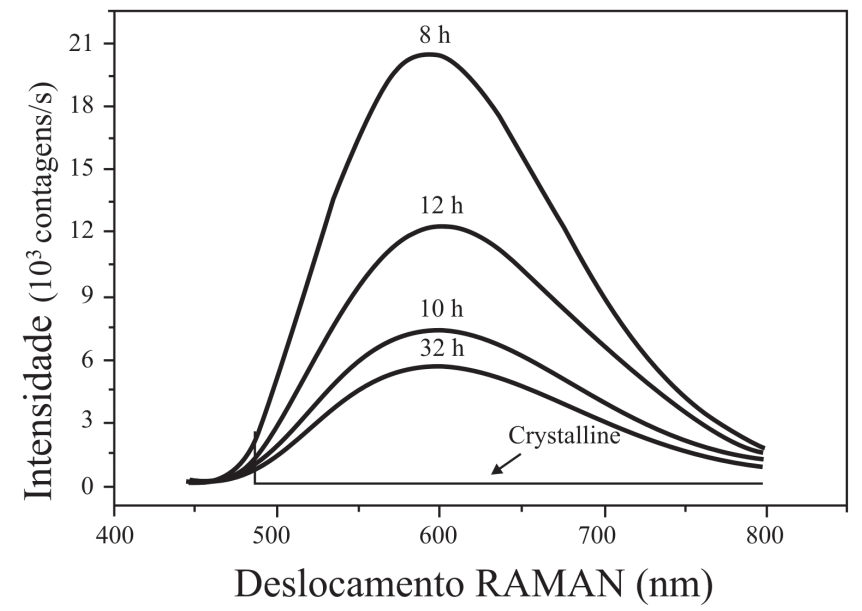

Figura 3: Espectro de FL do pó de $\mathrm{CaWO}_{4}$ amorfo, calcinado a 300 ${ }^{\circ} \mathrm{C}$ por períodos de tempo entre $8 \mathrm{~h}$ e $32 \mathrm{~h}$, e espectro do $\mathrm{CaWO}_{4}$ cristalino calcinado a $600{ }^{\circ} \mathrm{C} / 4 \mathrm{~h}$.

[Figure 3: Photoluminescence spectra of amorphous CaWO4 powders calcinated at $300{ }^{\circ} \mathrm{C}$ for different of time (8-32 h) and crystalline $\mathrm{CaWO}_{4}$ powder calcinated at $600^{\circ} \mathrm{C} / 4 \mathrm{~h}$.] 


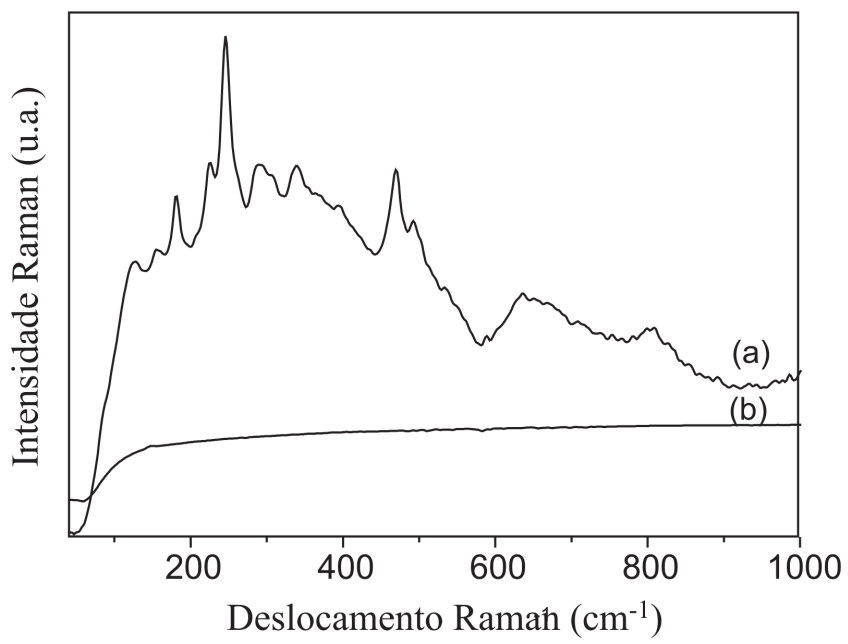

Figura 4: Espectros Raman para pó de $\mathrm{CaWO}_{4}$ a) cristalino e b) amorfo.

[Figure 4: Raman spectra of a) crystalline and b) amorphous $\mathrm{CaWO}_{4}$ powders.]

o material processado pelo método dos precursores poliméricos apresenta basicamente duas fases, uma inorgânica e outra fase constituída por carbono amorfo. Deste modo, se o comportamento da FL observado é devido à fase inorgânica desordenada, à medida que diminui o carbono pela queima em atmosfera oxidante, a FL aumenta. Este comportamento foi observado no espectro FL ilustrado na Fig. 3. Por outro lado, quando o material é calcinado a $600{ }^{\circ} \mathrm{C} / 4 \mathrm{~h}$ transformase na forma cristalina, daí a intensidade da FL reduzir drasticamente à temperatura ambiente e também na temperatura do nitrogênio líquido. Este comportamento foi observado na dependência do espectro de absorção em função da temperatura. Este fato é uma indicação forte de que a fase desordenada é responsável pelo fenômeno da FL [20-25]. Além disso, a Fig. 4 ilustra, na temperatura ambiente, o espectro Raman da fase cristalina e do amorfo. A predominância dos modos vibracionais ativos no espectro Raman indicam a presença da forma ortorrômbica da fase cristalina no $\mathrm{CW}$. Enquanto que no caso do $\mathrm{CW}$ amorfo, os picos do espectro desaparecem evidenciando que houve uma modificação nos parâmetros de rede, que associado ao resultado de difração de raios $\mathrm{X}$, caracteriza-o como sistema desorganizado.

$\mathrm{O} \mathrm{CW}$ amorfo mostrou uma dependência no espectro de absorção semelhante à apresentada em semicondutores amorfos (silício), enquanto o $\mathrm{CW}$ cristalino mostrou uma transição entre bandas, típica de materiais cristalinos. Estes resultados mostram que os dados estão consistentes com a interpretação na qual a extremidade de absorção óptica exponencial e o "gap" de banda óptico são controlados pelo grau de desordem, estrutural e térmico, na rede do composto CW. De outra maneira, estes resultados mostram que a absorção está associada aos novos estados localizado no "gap". Daí, a FL observada no semicondutor amorfo do CW foi ativada pela concentração dos buracos-elétrons devido a formação de defeitos. A Fig. 5 ilustra a dependência espectral de absorbância para um material amorfo $\mathrm{CW}$ tratado a $300^{\circ} \mathrm{C}$,

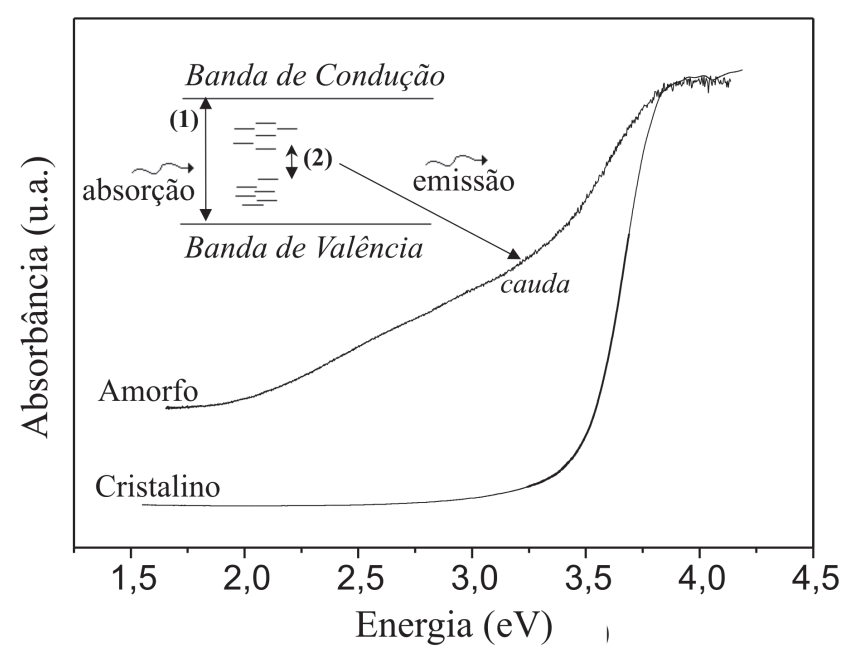

Figura 5: Espectro de absorção do pó de $\mathrm{CaWO}_{4}$ amorfo e cristalino: (1) energia da gap da banda para um processo de recombinacao direta, o que e impossível, (cristalino); (2) provável energia do gap da banda para o para o processo de recombinacao direta.

[Figure 5: Absorption spectra of amorphous and crystalline $\mathrm{CaWO}_{4}$ powders. The inset shows the scheme of the photoluminescence process in amorphous $\mathrm{CaWO}_{4}$ : (1) energy band gap for a direct recombination process, what is improbable (crystalline); (2) probable energy band gap for a recombination process.]

por $32 \mathrm{~h}$ na presença de oxigênio, e para o material cristalino do CW. O material amorfo do CW mostra uma dependência espectral de absorbância, tal como encontrada em semicondutores amorfos, tais como silício amorfo. Por outro lado, o material cristalino do $\mathrm{CW}$ mostrou um tipo de banda de material altamente organizado. Além disso, na região de alta energia da curva de absorbância (Fig. 5) o "gap" da banda de energia óptica é relacionado à absorbância e à energia do fóton pela seguinte equação:

$$
\text { hvo } \propto(\text { hv - Eopt })^{2} \text {, }
$$

em que h é a constante de Planck, $v$ é a freqüência e Eopt é o "gap" visual da banda [10]. As energias do "gap" da banda do material cristalino e do amorfo foram calculadas como 3,48 eV e 2,22 eV, respectivamente. Os resultados da Fig. 5 ilustram que os dados são consistentes com a interpretação da absorbância óptica, ou seja, o "gap" visível da banda é controlado pelo grau de desordem estrutural e térmica da amostra do CW.

Neste trabalho, o modelo está baseado na transferência de elétrons dos oxigênios para os íons $\mathrm{W}^{4+}[12,13]$. Assim sendo, os resultados obtidos nos cálculos teóricos indicam que na formação do "cluster" amorfo, por intermédio da deformação da ligação do oxigênio O(18), há introdução de níveis eletrônicos delocalizados na região entre a banda de valência e de condução. Estes novos níveis eletrônicos delocalizados são responsáveis pela formação da cauda no espectro de emissão (Fig. 6). A densidade parcial de estados do oxigênio estão presentes na banda de valência e do tungstênio e do cálcio 


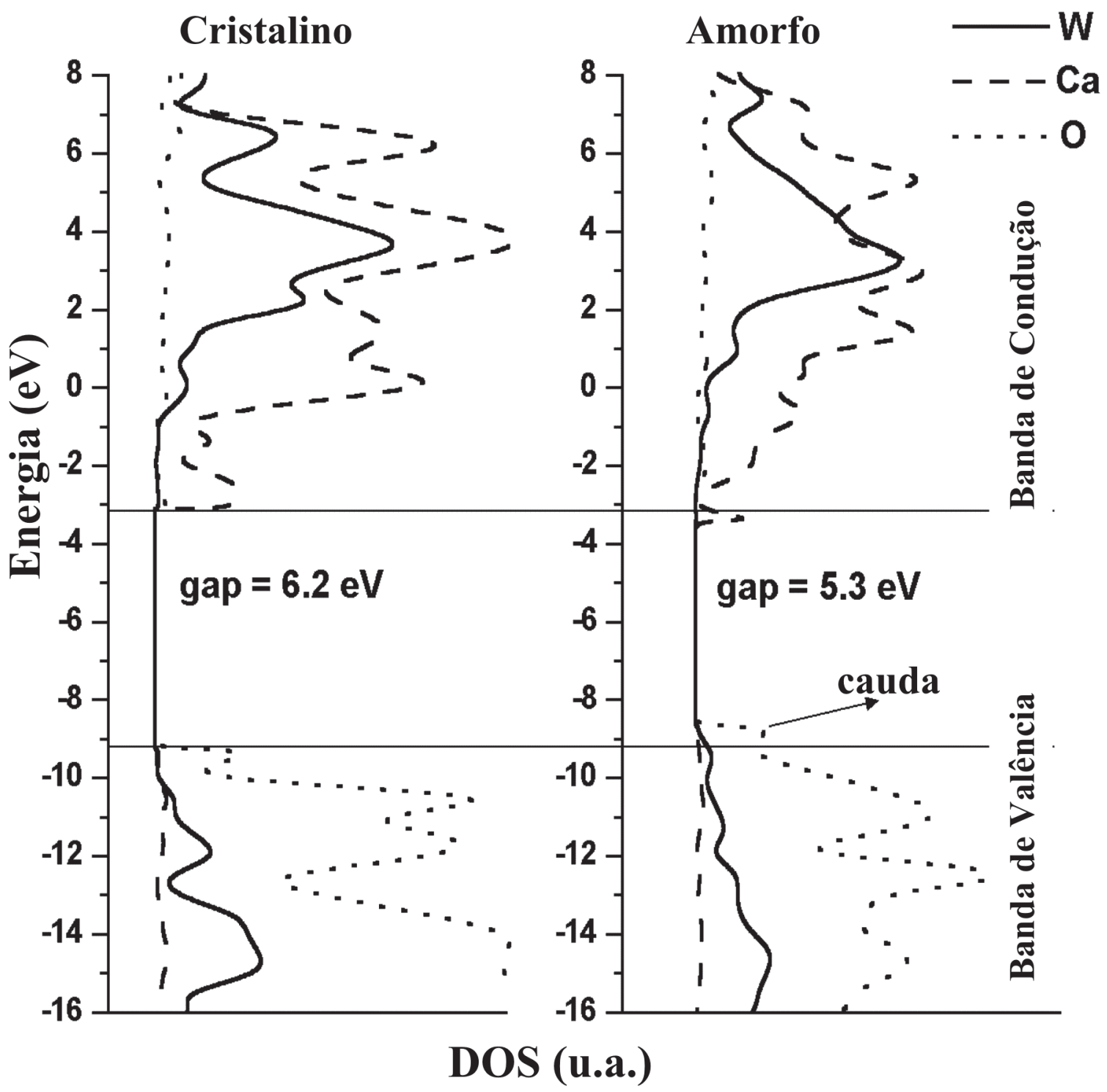

Figura 6: Representação da densidade parcial de estados do $\mathrm{Ca}_{4} \mathrm{~W}_{4} \mathrm{O}_{16}$, incluindo a banda de valência e a banda de condução. [Figure 6: Plot of partial densities of states for $\mathrm{Ca}_{4} \mathrm{~W}_{4} \mathrm{O}_{16}$, including the valence band and conduction band states.]

na banda de condução, conforme ilustrado na Fig. 6. Desta forma, nos "clusters" cristalino e amorfo existe uma contribuição pequena do cálcio e do tungstênio, para a densidade de estado na banda de valência, enquanto há uma forte contribuição do oxigênio. Entretanto, na parte superior da banda de condução, apresenta uma forte contribuição do tungstênio e do cálcio. O "cluster" cristalino apresenta um "gap" de banda superior ao do amorfo, em concordância com os resultados experimentais, observados nos espectros de absorbância. Os novos níveis eletrônicos criados pelos defeitos na banda de valência podem ser responsáveis, no material amorfo, pela propriedade FL. Os dados teóricos mostram que a deformação no "cluster" também conduz à formação de novos níveis eletrônicos na banda de valência. Da mesma forma, a banda de condução do $\mathrm{CW}$ é caracterizada, principalmente, pelo orbital $\mathbf{s}$ do cálcio e os orbitais $\mathbf{s}$ e $\mathbf{d}$ do tungstênio e a banda de valência que é constituída pelo orbital $\mathbf{p}$ oxigênio (Fig. 7). Assim sendo, os resultados dos cálculos teóricos indicam uma transferência de carga entre os dois centros dos $\mathrm{WO}_{4}$ (simétrico) e $\mathrm{WO}_{3}$ (assimétrico).

Na Fig. 7 estão ilustrados os níveis de energia e a distribuição de cargas nos orbitais moleculares para os íons de tungstênio e de cálcio. Neste caso, um íon de tungstênio forma um complexo tetraédrico $\mathrm{WO}_{4}$, e outro íon de tungstênio um trigonal deformado, constituído por um complexo $\mathrm{WO}_{3} \mathrm{e}$ uma vacância de oxigênio. Quando estas duas diferentes estruturas coexistem, há uma transferência de carga do "cluster" $\mathrm{WO}_{3}$ para o "cluster" $\mathrm{WO}_{4}$ caracterizando a formação de um buraco (h)-elétron (e').

Os cálculos teóricos sugerem que a formação dos dois "clusters" pode introduzir novos níveis eletrônicos em um "gap" proibido. Como consequiência, os orbitais p do oxigênio 

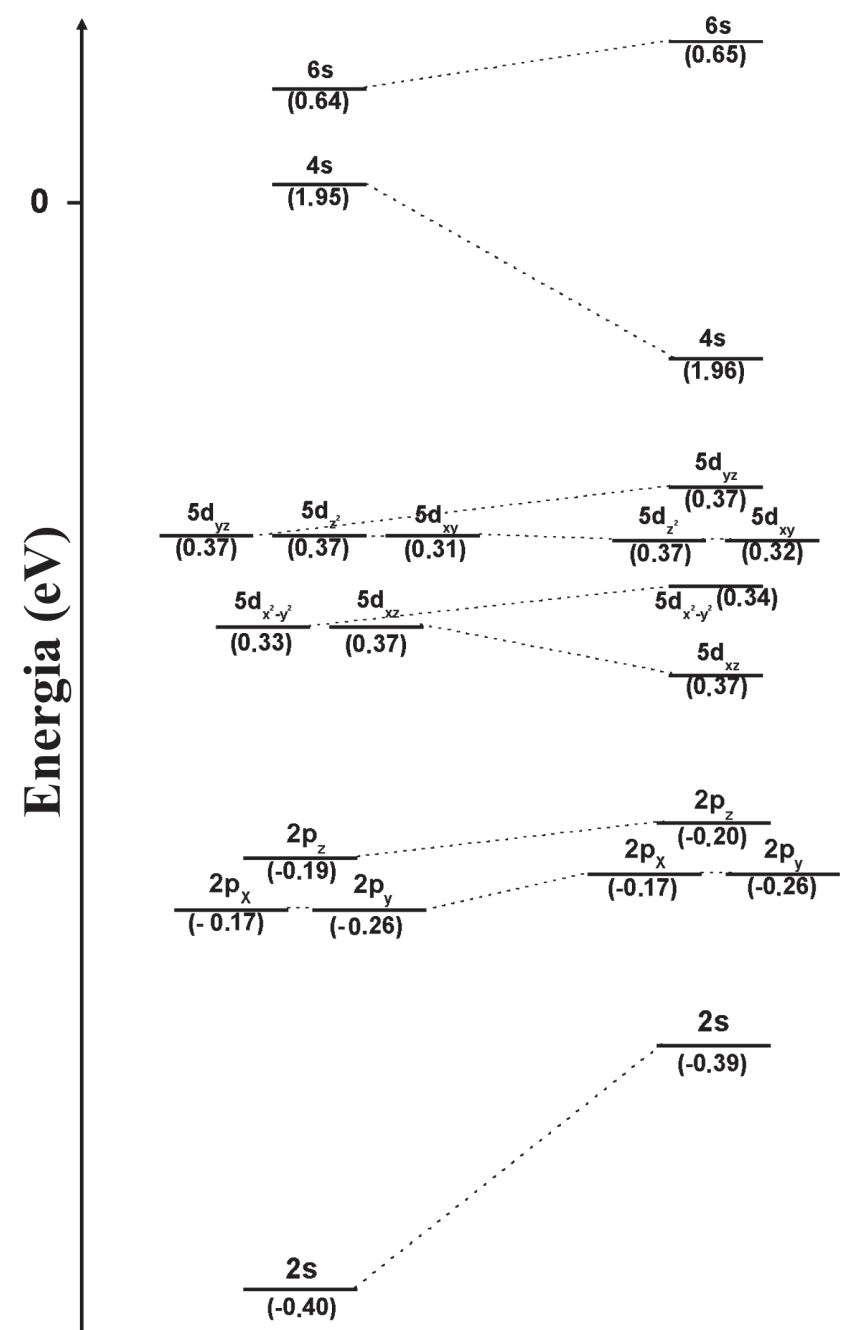

Figura 7: Diagrama esquemático de níveis de energia e distribuição da cargas nos orbitais moleculares do $\mathrm{Ca}_{4} \mathrm{~W}_{4} \mathrm{O}_{16}$, referente aos estados orbitais localizados na ligação $\mathrm{W}(4)-\mathrm{O}(18)$ do a) cristalino (c-CW) e b) amorfo (a-CW).

[Figure 7: Schematic diagram of the energy levels and charge distribution in the molecular orbital of $\mathrm{Ca}_{4} \mathrm{~W}_{4} \mathrm{O}_{16}$ in the W(4)-O(18) bond in (a) crystalline $(c-C W)$ and $(b)$ amorphous $(a-C W)$.]

são os principais responsáveis pela formação dos novos níveis na banda de valência.

Desta forma, a cauda observada corresponde aos defeitos na estrutura assimétrica. A geometria tetraédrica do "cluster" cristalino é favorecida, porque isso minimiza as interações eletrostáticas entre os quatro oxigênios ligantes arranjados em torno do tungstênio carregado positivamente (ver Fig. 1). Entre os orbitais d que formam ligações com os oxigênios existem as contribuições ligantes - anti-ligantes. (Fig. 6). Conceitualmente, o "cluster" amorfo foi obtido do "cluster" cristalino pelo prolongamento da ligação $\mathrm{O}(18)-\mathrm{W}(4)\left[\mathrm{WO}_{3}\right]$. Com esta deformação, o orbital anti-ligante $3 \mathbf{d x z}$ será estabilizado no "cluster" $\mathrm{WO}_{3}$, porque existe somente uma interação anti-ligante, com relação às duas que estão presentes no $\mathrm{WO}_{4}$. Assim sendo, os resultados teóricos indicam que a formação do $\mathrm{WO}_{3}$ tri-coordenado, pelo deslocamento do $\mathrm{O}(18)$ e podendo introduzir deformação de níveis eletrônicos acima do HOMO do "cluster" cristalino, com os orbitais $2 \mathbf{p}_{\mathrm{x}}, 2 \mathbf{p}_{\mathrm{y}} \mathrm{e}$ $2 \mathbf{p}_{\mathrm{z}}$ do oxigênio. Isto significa que a combinação dos ligantes dxz no "cluster" cristalino é instável, e no "cluster" amorfo é estável. Consequentemente, o "gap" formado pelos orbitais $\mathbf{s}$ e dxz do tungstênio e $\mathbf{s}$ do cálcio no (LUMO) e os orbitais $\mathbf{p}$ do oxigênio (HOMO), é maior para o "cluster" cristalino, do que no "cluster" amorfo. Neste caso, o tungstênio e o cálcio são fortemente favorecidos para uma configuração mais estável para orbitais d e s do "cluster" $\mathrm{WO}_{3}$ (Fig. 7).

As interações entre $\mathrm{W}$ e $\mathrm{O}$ no complexo $\mathrm{WO}_{4}$ são fortes comparativamente à do complexo $\mathrm{WO}_{3}$. Deste modo, a combinação de ligações entre os orbitais $d_{z} 2$ e $d_{x} 2-y$ no "cluster" simétrico é instável e no "cluster" assimétrico é estável (Fig. 7). Como conseqüência, o gap entre o orbital $\mathrm{d}(\mathrm{W})$ (LUMO) e p(O) (HOMO) é maior para o "cluster" simétrico comparativamente ao gap para o "cluster" assimétrico. Em adição, o "cluster" cristalino tem níveis de energia no orbital estável d(W) e p(O) e a distribuição de carga ligeiramente maior na ligação W(4)-O(18). Por outro lado, o "cluster" assimétrico tem níveis de energia no orbital d do W(4) marcantemente mais altos e distribuição de carga ligeiramente mais baixa. A partir destes resultados é aparente que o topo da banda de valência contribui a partir do orbital $\mathbf{p}$ do oxigênio.

Os cálculos de Ab-initio sugerem que o fenômeno da fotoluminêscencia é resultado da recombinação dos elétrons e par de buracos polarons, devido a transferência de carga entre os "clusters" $\mathrm{WO}_{4}$ e $\mathrm{WO}_{3}$.

\section{CONCLUSÕES}

Para os materiais de $\mathrm{CW}$ estudados neste artigo, os resultados das análises da densidade de estados, juntamente com os resultados experimentais, concordam qualitativamente que os defeitos na estrutura são responsáveis pelas propriedades FL. Os resultados experimentais mostraram que a emissão FL visível no amorfo à temperatura ambiente está diretamente relacionada com a extremidade de absorção óptica exponencial. A natureza desse limite óptico exponencial pode estar associada com as deformações promovidas pela desordem estrutural do material amorfo. Pelos cálculos utilizando os primeiros princípios, acredita-se que a emissão visível não pode ser atribuída à transição banda-a-banda do $\mathrm{CW}$, porque a linha de energia de excitação usada $(488,0 \mathrm{~nm} / 2,52 \mathrm{ev})$ no experimento é muito mais curta do que o gap da banda do cristal CW (3,48 ev). Como consequiência, é impossível excitar diretamente o elétron do orbital $2 \mathrm{p}$ do oxigênio, para o orbital 4 s do cálcio e o $3 \mathrm{~d}$ do tungstênio. A banda de condução do material amorfo é formada pelos orbitais do cálcio e tungstênio, enquanto que a banda de valência é principalmente composta pelos orbitais sp do oxigênio. Além disso, os resultados dos cálculos teóricos indicam a formação da espécie tricoordenada por intermédio do deslocamento do oxigênio $\mathrm{O}(18)$. Estas 
deformações, ou centros de defeitos que existem no material amorfo do CW, geram novos níveis eletrônicos na região do gap da banda (banda proibida) que, por sua vez, podem atuar como centros de absorção óptica responsáveis pela fotoluminêscencia visível à temperatura ambiente.

Os cálculos teóricos suportam o modelo da fotoluminêscencia no $\mathrm{CaWO}_{4}$ amorfo como um resultado da recombinação de elétrons e buracos polarons resultante da transferência de carga entre os "clusters" $\mathrm{WO}_{4}$ e o $\mathrm{WO}_{3}$.

\section{AGRADECIMENTOS}

Os autores agradecem a FAPESP/CEPID, CNPq/ PRONEX e CAPES pelo suporte financeiro desta pesquisa.

\section{REFERÊNCIAS}

[1] L. T. Canham, Appl. Phys. Lett. 57 (1990) 1046.

[2] J. Ballato, R. Esmacher, R. Schwartz, M. Dejneka, J. Luminescence 86 (2000) 101.

[3] H. Liu, S. T. Li, G. K. Liu, W. Jia, F. E. Fernández, J. Luminescence 83-84 (1999) 367.

[4] V. Trapakov, V. Dimza, L. Jastrabik, A. Savinov, Z. Bryknar, Phys. Stat. Solidi 183 (1994) 299.

[5] Z. Bryknar, V. Trepakov, Z. Potucek, L. Jastrabik , J. Luminescence 87 (2000) 605.

[6] S. Murakami, M. Morita, M. Herren, T. Sakurai, D. Rau, J. Luminescence 87-89 (2000) 694.

[7] W. F. Zhang, Z. Yin, M. S. Zhang, Appl. Phys. A 70 (2000) 93. [8] J. Meng, Y. Huang, W. Zhang, Z. Du, Z. Zhu, H. Zou, Phys. Lett. A 205 (1995) 72.
[9] B. Bouma, G. Blasse, J. Phys. Chem. Solids 56 (1995) 261. [10] D. L. Wood, J. Tauc, Phys. Rev. B 5 (1972) 3144.

[11] M. Capizzi, A. Frova, Phys. Rev. Lett. 25 (1970) 1298.

[12] P. S. Pizani, E. R. Leite, F. M. Pontes, E. C. Paris, J. H. Rangel, E. Lee, E. Longo, P. Delega, J. A Varela, Appl. Phys. Lett. 77 (2000) 824.

[13] F. M. Pontes, E. R. Leite, E. Longo, J. A. Varela, P. S. Pizani, C. E. M. Campos,

F. Lanciotti, Adv. Mater. Opt. Electron. 10 (2000) 81.

[14] F. M. Pontes, E. Longo, E. R. Leite, J. A. Varela, Thin Solid Films 386 (2001) 91.

[15] F. M. Pontes, E. B. Araújo, E. R. Leite, J. A. Eiras, E. Longo, J. A. Varela, Appl. Phys. Lett. 76 (2000) 2433.

[16] E. R. Leite, I. T. Weber, E. Longo, J. A. Varela, Adv. Mater. 12 (2000) 965.

[17] P. C. Harihara, J. A. Pople, Theor. Chim. Acta 28, 3 (1973) 213.

[18] P. J. Hay, W. R. Wadt, J. Chem. Phys. 82, 1 (1985) 270.

[19] M. J. Frisch et al., Gaussian 98, Revision A.6, Gaussian, Inc., Pittsburgh, PA, (1998).

[20] J. P. Foster, F. Weinhold, J. Am. Chem. Soc. 102, 24 (1980) 7211.

[21] A. E. Reed, L. A. Curtiss, F. Weinhold, Chem. Rev. 88, 6 (1988) 899.

[22] A. E. Reed, R. B. Weinstock, F. Weinhold, J. Chem. Phys. 88, 2 (1985) 735.

[23] V. Balanchandran, N. G. Eror, J. Solid State Chem. 39 (1981) 351.

[24] N. H. Chan, R. K. Sharma, D. M. Smyth, J. Electrochem. Soc. 128 (1981) 1762.

[25] A. H. Kahn, A. J. Leyendecker, Phys. Ver. A 135 (1964) 1321.

(Rec. 20/08/03, Ac. 31/10/03) 